\title{
Retroperitoneal mucinous cystadenoma of the appendix mimicking hydatid cyst: A case report
}

\author{
HASAN EDIZ SIKAR ${ }^{1}$, KENAN ÇETIN ${ }^{1}$, ERSIN GÜNDOĞAN ${ }^{1}$, \\ GÖKÇEN ALINAK GÜNDOĞAN ${ }^{2}$ and LEVENT KAPTANOĞLU ${ }^{1}$
}

\begin{abstract}
Departments of ${ }^{1}$ General Surgery and ${ }^{2}$ Pathology, Dr Lütfi Kirdar Kartal Research and Education Hospital, Istanbul, Turkey
\end{abstract}
Received November 20, 2015; Accepted June 2, 2016

DOI: $10.3892 / \operatorname{mco} .2016 .921$

\begin{abstract}
Appendiceal mucocele is a cystic dilatation of the appendix due to abnormal appendiceal mucinous secretion. Cystadenoma of the appendix is one of the most common causes and is encountered in $0.6 \%$ of all appendectomy specimens. The diagnosis may be difficult due to the asymptomatic nature of the disease; pain in the right lower quadrant may be the only symptom. Complex ovarian cyst, urolithiasis or cystic hydatid disease of the liver have been reported as mimicking appendiceal mucocele in the literature. In this study, we present a case of mucinous cystadenoma of the appendix mimicking retroperitoneal hydatid cyst in a 59-year-old woman. The patient was treated with laparoscopic appendectomy with partial resection of the caecum following laparoscopic exploration.
\end{abstract}

\section{Introduction}

Appendiceal mucocele results from obstruction of the appendiceal orifice and cystic dilatation of the appendiceal lumen due to mucinous secretions (1). Appendiceal mucocele may be inflammatory or have a neoplastic origin. Primary neoplastic lesions presenting with a mucocele of the appendix are rare. Mucoceles may occur as a result of a retention cyst, mucosal hyperplasia, mucinous cystadenoma or mucinous cystadenocarcinoma. Cystadenoma of the appendix is one of the most common causes and may be detected in $0.6 \%$ of appendectomy specimens (2). In the majority of the patients, asymptomatic; pain in the right lower quadrant of the abdomen may be the only symptom. Preoperative diagnosis is difficult and patients are often diagnosed incidentally by abdominal surgery for other causes $(3,4)$.

Correspondence to: Dr Hasan Ediz Sikar, Department of General Surgery, Dr Lütfi Kirdar Kartal Research and Education Hospital, Main Building, First Floor, Cevizli, Kartal, Istanbul, Turkey

E-mail: hasan.sikar@me.com

Abbreviations: US, ultrasonography; CT, computed tomography

Key words: mucocele, mucinous cystadenoma, echinococcosis
We herein present a case of a female patient who was misdiagnosed with retroperitoneal hydatid cyst and underwent laparoscopic appendectomy with partial resection of the caecum following laparoscopic exploration.

\section{Case report}

A 59-year old female patient was admitted to the Dr Lütfi Kirdar Kartal Research and Education Hospital (Istanbul, Turkey) with right upper quadrant abdominal pain for 3 years. The physical examination and laboratory findings revealed no abnormalities. Abdominal ultrasonography (US) revealed a retroperitoneal cystic mass with low-level internal echoes, suspicious for hydatid disease, in the right upper quadrant. A computed tomography scan $(\mathrm{CT})$ revealed a retroperitoneal cystic mass, sized $11 \times 6 \mathrm{~cm}$, with partial calcifications of the wall, consistent with the characteristics of echinococcal hydatid cyst (Fig. 1). Serodiagnostic markers were within the normal range, but the patient resided in a part of Turkey endemic for echinococcosis.

Laparoscopic cystectomy was planned. Three trocars were placed in the left lateral decubitus position, as applied during adrenalectomy procedures. The paracolic peritoneum was divided and posterior attachments with the kidney and duodenum were released. With the help of a gauze pad, the cyst was manipulated carefully to avoid perforation. Laparoscopic exploration revealed a giant appendiceal mucocele. The caecum, including the base of the appendix, was partially resected with an endoscopic stapler and the specimen was extracted with an endobag (Fig. 2).

The histopathological examination revealed an appendix sized 11x6 cm, filled with mucin. The definitive diagnosis was mucinous cystadenoma of the appendix and the surgical margins were clear behind the staple line (Fig. 3). The postoperative recovery was uneventful and the patient was discharged on postoperative day 3. Colonoscopy and US were performed 3 months after surgery for the possibility of metachronous cancer and pseudomyxoma peritonei, respectively, with no pathological findings.

\section{Discussion}

The term appendiceal mucocele refers to the cystic dilatation of the appendiceal lumen caused by mucinous secretions (1). 
Appendiceal mucocele may develop due to mucinous cystadenoma (63\%), mucosal hyperplasia (25\%), mucinous cystadenocarcinoma (11\%) and retention cyst of the appendiceal lumen (1\%) (3). Appendiceal mucocele is more frequent in women aged $>50$ years. The most common cause of appendiceal mucocele is mucinous cystadenoma, which is detected in $0.6 \%$ of appendectomy specimens (2). Preoperative diagnosis is difficult, as $\sim 25-50 \%$ of the patients are asymptomatic and diagnosed incidentally during physical examination or during abdominal imaging or surgery (4). Symptomatic patients present with acute or chronic right lower quadrant pain. Other symptoms, such as nausea, vomiting or weight loss, may point towards mucinous cystadenocarcinoma or pseudomyxoma peritonei.

Abdominal US and CT scan may be helpful for diagnosis. A round, ovoid, sausage-, pear- or chicken drumstick-shaped cystic mass, with a variable intraluminal echotexture and low-level internal echoes or septae are diagnostic findings on US $(4,5)$. CT scan is superior to US for evaluation of the appendix, as it shows the topographic anatomical associations between the caecum and the mucocele. In addition, CT scan is more sensitive in the detection of mural curvilinear calcifications compared with US.

Hydatid disease is a zoonosis and generally caused by infection with Echinococcus granulosus. The most common type of disease appearance is cysts, and the liver is the most common site (6). Although retroperitoneal hydatid disease is a very rare condition, the eastern region of Turkey is an endemic area for echinococcosis and cystectomy is the treatment of choice for preventing spread and recurrence. The serodiagnosis was negative, but the sensitivity and specificity of serodiagnostic tests for echinococcosis have been reported to range between 80 and $90 \%$ (7). False-negative results in our daily practice increased the significance of imaging for diagnosis and led to a revision of treatment options. Cystectomy or pericystectomy is our choice of treatment, rather than puncture-aspiration-irrigation-reaspiration or partial cystectomy, in cases highly suspicious for hydatid disease but with negative serodiagnosis. Complex ovarian cyst, urolithiasis or cystic hydatid disease of the liver have been reported to mimick appendiceal mucocele based on US or CT findings and non-specific clinical symptoms $(3,4,8)$. To the best of our knowledge, there are no reports of mucinous appendiceal neoplasms presenting as retroperitoneal hydatid cysts.

Spontaneous or intraoperative rupture of the mucocele and spread of epithelial cells and mucoid fluid throughout the peritoneal cavity, resulting in pseudomyxoma peritonei, is the most serious complication of appendiceal mucocele (1). Although some authors do not recommend laparoscopic treatment for appendiceal mucocele, the laparoscopic approach is suitable when performed carefully. Every effort is made to maintain the mucocele intact; direct manipulation of the mucocele may result in rupture and should be avoided; a gauze pad must be used to manipulate the appendiceal mucocele (9-11). Extracting the specimen with an endobag is mandatory.

It is generally accepted that appendectomy is suitable for unruptured benign mucoceles localized to the appendix $(1,11)$. There is no consensus on the surgical treatment for mucoceles involving the base of the appendix or invaginating into the caecum. Right hemicolectomy, ileocaecal resection and

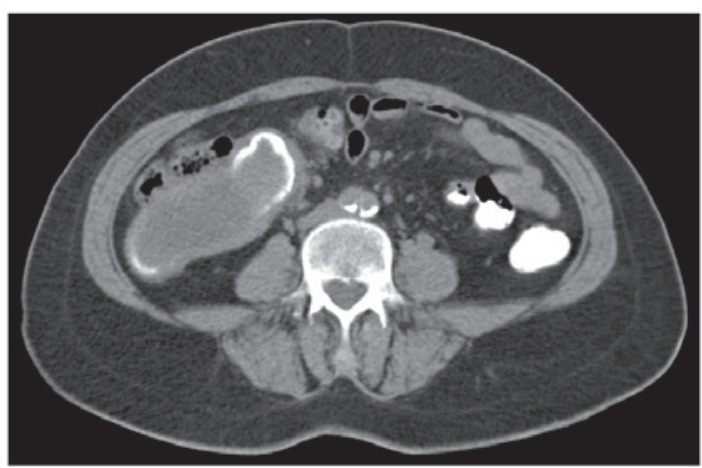

Figure 1. Computed tomography scan showing a retroperitoneal cystic mass with partial calcifications on the wall.

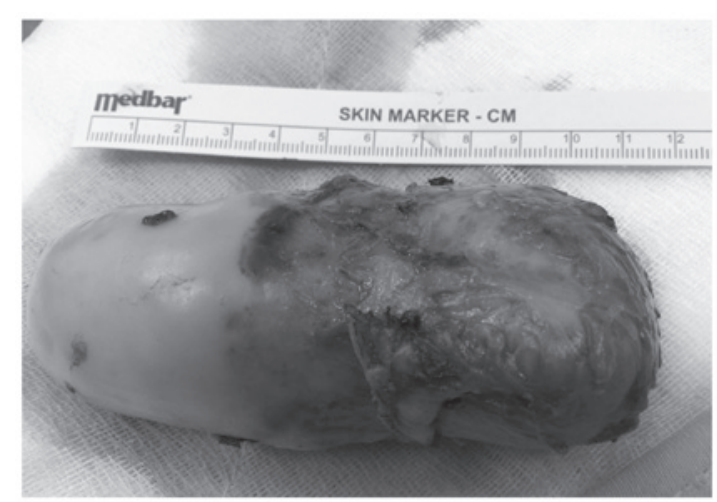

Figure 2. Macroscopic specimen of the appendiceal mucocele including the base of the appendix.

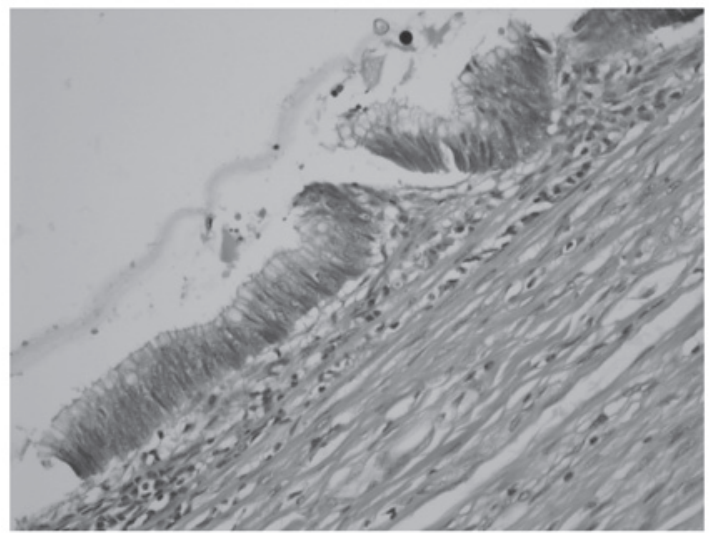

Figure 3. Microscopic view of the mucocele, showing a single row of columnar mucinous epithelium-lined cyst wall (hematoxylin and eosin staining; magnification, x200).

appendectomy with partial resection of the caecum have been evaluated as therapeutic modalities $(1,4,8,11)$. The postoperative period in patients undergoing appendectomy with partial resection of the caecum is generally uneventful $(8,11)$. Due to the higher morbidity rate of right hemicolectomy, appendectomy with partial resection of the caecum is the preferred treatment in selected cases.

The postoperative follow-up of patients is important, due to the high incidence of synchronous or metachronous 
cancer $(20 \%)$ (11). Colonoscopy should be performed after recovery for close follow-ups; it is also recommended for preoperative diagnosis. In addition, abdominal US is important for the detection of postoperative pseudomyxoma peritonei caused by unnoticed contamination.

In conclusion, appendiceal mucocele should be considered when an atypical cystic mass is identified in the right upper or lower quadrants of the abdomen by US or CT scan. The laparoscopic approach is suitable when performed carefully. In cases highly suspicious for hydatid disease with negative serodiagnosis, cystectomy is the treatment of choice for the prevention of spread and recurrence.

\section{References}

1. Maa J and Kirkwood KS: The appendix. In: Sabiston Textbook of Surgery. Townsend CM, Beauchamp RD, Ever BM and Mattox KL (eds.) Elsevier Saunders, Philadelphia, pp1279-pp1293, 2012.

2. Marudanayagam R, Williams GT and Rees Bl: Review of the pathological results of 2660 appendicectomy specimens. J Gastroenterol 41: 745-749, 2006.

3. Demirci RK, Habibi M, Karakaş BR, Buluş H, Akkoca M and Öner OZ: Appendix mucocele mimicking a complex ovarian cyst. Ulus Cerrahi Derg 31: 58-60, 2013.
4. Krieg A, Esch JS II, Poll LW, Braunstein S and Knoefel WT: Mucinous cystadenoma of the appendix misdiagnosed as cystic hydatid disease of the liver: A case report. J Med Case Rep 2: 218, 2008.

5. Madwed D, Mindelzun R and Jeffrey RB Jr: Mucocele of the appendix: Imaging findings. AJR AM J Roentgenol 159: 69-72, 1992.

6. Cakir OO, Ataseven $\mathrm{H}$ and Demir A: Hydatid acute pancreatitis. Turkiye Parazitol Derg 36: 251-253, 2012 (In Turkish).

7. Siracusano A, Buttari B, Delunardo F, Profumo E, Margutti P, Ortona $\mathrm{E}$, Riganò $\mathrm{R}$ and Teggi $\mathrm{A}$ : Critical points in the immunodiagnosis of cystic echinococcosis in humans. Parassitologia 46: 401-403, 2004

8. Dandin Ö, Balta AZ, Sücüllü I, Yucel E, Ozgan ET and Yıldırım Ş: Appendiceal mucocele mimicking urolithiasis. Ulus Cerrahi Derg 29: 88-91, 2013.

9. Rangarajan M, Palanivelu C, Kavalakat AJ and Parthasarathi R: Laparoscopic appendectomy for mucocele of the appendix: Report of 8 cases. Indian J Gastroenterol 25: 256-257, 2006.

10. Navarra G, Asopa V, Basaglia E, Jones M, Jiao LR and Habib NA: Mucous cystadenoma of the appendix: Is it safe to remove it by a laparoscopic approach? Surg Endosc 17: 833-834, 2003

11. Kılıç MÖ, İnan A and Bozer M: Four mucinous cystadenoma of the appendix treated by different approaches. Ulus Cerrahi Derg 30: 97-99, 2014. 